\title{
Intravitreal Ranibizumab versus Isovolemic Hemodilution in the Treatment of Macular Edema Secondary to Central Retinal Vein Occlusion: Twelve-Month Results of a Prospective, Randomized, Multicenter Trial
}

\author{
Thomas C. Kreutzer ${ }^{a, e}$ f Armin Wolf ${ }^{a}$ Martin Dirisamer ${ }^{e, f}$ Rupert W. Strauss ${ }^{e}$ \\ Paul Foerster $^{a}$ Nicolas Feltgen ${ }^{b}$ Amelie Pielen ${ }^{c}$ Lars-Olof Hattenbach ${ }^{d}$ \\ Anselm Kampik $^{a}$ Siegfried G. Priglinger ${ }^{a}, \mathrm{e}, \mathrm{f}$ RAVO Study Group \\ a University Eye Hospital, Ludwig Maximilian University, Munich, ${ }^{b}$ University Eye Hospital, Georg August \\ University, Göttingen, ' University Eye Hospital, Albert Ludwig University Hospital, Freiburg, and dDepartment of

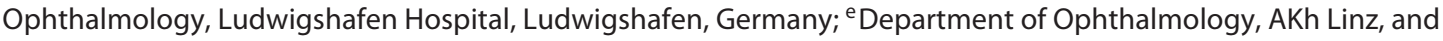 \\ ${ }^{f}$ Medical Faculty, Johannes Kepler University, Linz, Austria
}

\section{Key Words}

Central retinal vein occlusion $\cdot$ Hemodilution $\cdot$ Randomized trial · Ranibizumab · Vascular endothelial growth factor

\footnotetext{
Abstract

Purpose: This is a prospective, randomized, multicenter, investigator-initiated trial to evaluate the 12-month effectiveness of isovolemic hemodilution $(\mathrm{IH})$ with prompt versus deferred intravitreal injections (IVI) of ranibizumab $0.5 \mathrm{mg}$ for the treatment of macular edema secondary to early central retinal vein occlusion (CRVO). Methods: Eyes with macular edema due to CRVO having occurred not more than 8 weeks previously received either monthly ranibizumab IVI in combination with $\mathrm{IH}$ (group I, $\mathrm{n}=28$ ) or $\mathrm{IH}$ alone (group II, $\mathrm{n}=30$ ). From month 2 to 12 , the patients in both groups could be treated with monthly intravitreal ranibizumab. The main outcome variables were gain of visual acuity and the course of central retinal thickness as measured with optical coherence tomography. Results: At 12 months, eyes in group I on average gained $+28.1( \pm 19.3)$ letters compared to +25.2
}

$( \pm 20.9)$ letters in group II $(p=0.326)$. This result was achieved with significantly fewer injections in group II. Additionally, $30 \%$ of the eyes in group II did not need ranibizumab IVI during the 12 months of the trial. Conclusion: Ranibizumab IVI in addition to $\mathrm{IH}$ proved to be highly effective in increasing visual acuity and reducing macular edema secondary to CRVO. Initial IH in early CRVO may be a first treatment option in patients anxious about IVI.

(c) 2014 S. Karger AG, Basel

\section{Introduction}

Central retinal vein occlusion (CRVO) is the second most frequent retinal vascular disease with a prevalence between 0.3 and $0.6 \%$ [1]. For decades, treatment was largely based on the results of the Central Vein Occlusion

The trial protocol No. is LUZVT06/RAVO-Studie, EudraCT 2006005450-71.

\section{KARGER 125}

C 2014 S. Karger AG, Base

$0030-3755 / 14 / 2331-0008 \$ 39.50 / 0$

E-Mail karger@karger.com

www.karger.com/oph
Siegfried G. Priglinger, MD

Department of Ophthalmology

AKh Linz, Krankenhausstrasse 9

AT-4021 Linz (Austria)

E-Mail siegfried.priglinger@akh.linz.at 
Study [2]. As a result, only panretinal laser photocoagulation in pronounced ischemic cases proved to prevent further complications, but it did not improve visual recovery [3]. In the early 1980s, Hansen et al. [4] showed favorable results concerning visual acuity in CRVO when treated with isovolemic hemodilution (IH) which was initiated early (duration of symptoms $<8$ weeks). Similar results have also been described by Glacet-Bernard et al. $[5,6]$. Though based on a well-performed clinical trial, due to the small study sample, their IH results did not find worldwide acceptance as a general treatment regimen in CRVO [7]. In Germany, IH is commonly considered a primary treatment option [8].

In recent years, intravitreal pharmaceutical blockage of vascular endothelial growth factor (VEGF) has shown efficacy in the treatment of CRVO [9-11]. Early noncomparative clinical trials using intravitreal bevacizumab (Avastin $^{\mathrm{TM}}$; Roche Pharma AG, Grenzach-Wyhlen, Germany), a monoclonal antibody binding and deactivating VEGF molecules, found favorable results of visual acuity recovery in CRVO. Primarily developed as an intravenous treatment of gastrointestinal cancer, the therapy remained off label. Ranibizumab (Lucentis ${ }^{\mathrm{TM}}$; Novartis Pharma GmbH, Basel, Switzerland), a smaller antibody with the same binding region developed and approved for the intravitreal treatment of age-related macular degeneration, was efficacious in a prospective, randomized, clinical trial in CRVO patients up to 24 months. Approval for macular edema in central and branch retinal vein occlusion followed in 2011. In the CRUISE study, patients treated with monthly ranibizumab $0.5 \mathrm{mg}$ improved by 14.9 letters at month 6 in contrast to 0.8 letters in the control group [12]. In 2007, the RAVO study, a multicenter, prospective, randomized trial in Germany, was initiated to evaluate the effectiveness of intravitreal treatment with ranibizumab $0.5 \mathrm{mg}$ in combination with $\mathrm{IH}$ compared to $\mathrm{IH}$ alone followed by ranibizumab $0.5 \mathrm{mg}$ in a pro re nata (PRN) regimen for patients with macular edema due to fresh CRVO (duration of symptoms $<8$ weeks). The main outcome parameters of this study were gain of visual acuity, the course of central retinal thickness (CRT) and the number of injections.

\section{Methods}

The RAVO trial is an open, prospective, randomized, controlled, multicenter, investigator-initiated clinical trial to evaluate the 12-month effectiveness of IH with prompt versus deferred intravitreal injections (IVI) of ranibizumab $0.5 \mathrm{mg}$ for the treatment of macular edema secondary to early CRVO.

IV Ranibizumab vs. IH in the Treatment of Macular Edema Secondary to CRVO
The study was performed in accordance with the tenets of the Declaration of Helsinki. All patients gave their informed consent prior to any study-related procedures. The study was accepted by the ethics committees at each study site as well as by the federal authorities. This trial is registered under EudraCT No. 2006005450-71.

The RAVO study aimed at enrolling 60 patients (30 per group) with macular edema secondary to CRVO. Only one eye per patient could be included. The main inclusion and exclusion criteria are summarized in table 1. Only treatment-naive patients with CRVO over a period of no more than 8 weeks were included.

\section{Primary and Secondary Endpoints}

The primary endpoints were visual acuity results on the Early Treatment Diabetic Retinopathy Study (ETDRS) chart and macular edema after 8 weeks ( 2 months). The secondary endpoints were visual acuity results and macular edema after 52 weeks ( 12 months). Additional endpoints were percentages of patients gaining $\geq 3$ lines on the ETDRS chart, the number of injections and treatment safety after 12 months.

At every visit, visual acuity was tested according to the ETDRS guidelines, CRT was measured with a Stratus OCT-3000 device (Carl Zeiss Meditec, Dublin, Calif., USA) via a fast macular scan, fundus photographs were taken of the central temporal $30^{\circ}$, and a full ophthalmic evaluation with dilated fundus biomicroscopy was performed. At baseline as well as at months 2, 6 and 12, fluorescein angiography was performed. Blood pressure measurements and blood samples were taken at baseline and at every visit during the first 8 weeks. The blood examination consisted of a blood count, hematocrit and coagulation parameters (international normalized ratio and partial thromboplastin time). At baseline, the erythrocyte sedimentation rate as well as C-reactive protein and serum creatinine levels were acquired.

\section{Treatment}

Treatment in both groups consisted of IH starting within 3 days of enrollment. IH was performed by full venous blood extraction of $500 \mathrm{ml}$ into a blood donation bag from one arm and simultaneous venous infusion of $500 \mathrm{ml} 6 \%$ hydroxyethyl starch by way of the contralateral arm [13]. This procedure was repeated within the first week until a hematocrit level of $36 \%$ was reached. If hematocrit levels during the first 8 weeks rose above $38 \%$, IH was repeated. Visits were scheduled every 2 weeks during the first 8 weeks and then on a 4-weekly basis. In group I, the patients additionally received monthly IVI of ranibizumab $0.5 \mathrm{mg}$, initiated within the first week of enrollment on a PRN basis. In both groups, the patients received IVI of ranibizumab $0.5 \mathrm{mg}$ on a PRN basis from week 8 onwards if the following criteria for injection were met: visual acuity $<20 / 25$ and/or CRT $>225 \mu \mathrm{m}$ on optical coherence tomography (OCT). In both groups, IVI were discontinued if the following criteria were met: visual acuity $>20 / 25$ and/or CRT $<225$ $\mu \mathrm{m}$ on OCT.

\section{IVI Technique}

Before each scheduled IVI, the patients received polymyxin B, neomycin and bacitracin eyedrops 4 times for 3 days (Polyspectran; Alcon Pharma GmbH, Freiburg, Germany). Topical anesthesia before IVI was performed at least 3 times using tetracaine $1 \%$ eyedrops. Before injection, the brow, upper and lower eyelids and eyelashes as well as adjacent forehead, nose, cheek and temporal 
Table 1. Inclusion and exclusion criteria of the RAVO study

Inclusion criteria

Best-corrected visual acuity at baseline in the study eye between 5 and 65 letters using an ETDRS chart measured at $4 \mathrm{~m}$ or at $1 \mathrm{~m}$

Cystoid macular edema secondary to CRVO of $\geq 250 \mu \mathrm{m}$

Maximum duration of symptoms: 8 weeks

Willingness and ability to follow the study protocol

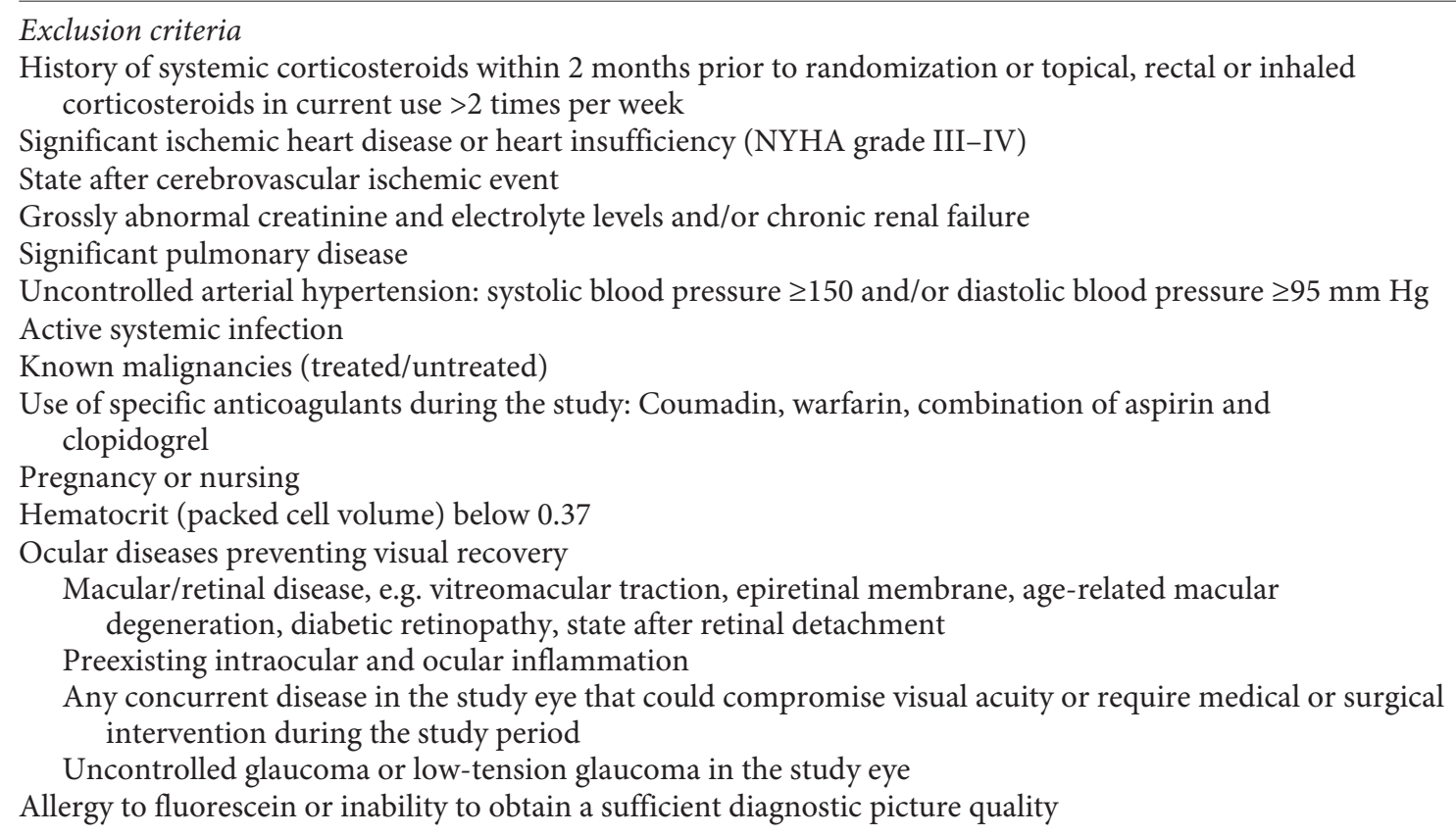

orbital areas were scrubbed with povidone-iodine $10 \%$, and the conjunctiva was irrigated with $10 \mathrm{ml}$ of povidone-iodine $1 \%(\mathrm{Be}-$ tadine; Alcon, Fort Worth, Tex., USA) through the upper and lower fornices. After application of a sterile drape, a lid speculum was inserted. The patients received 1 unilateral IVI (ranibizumab 0.5 $\mathrm{mg} / 0.05 \mathrm{ml}$ ) using a sharp 27-gauge needle at a distance of 3.5 and $4.0 \mathrm{~mm}$ from the limbus in pseudophakic and phakic eyes, respectively. The needle was carefully removed using a sterile cotton applicator to prevent reflux. After the injection, the patients were asked to continue to self-administer antibiotic eyedrops (polymyxin $\mathrm{B}$, neomycin and bacitracin) for 3 more days 4 times per day. The drug $(0.1 \mathrm{ml})$ was drawn under sterile conditions from a ranibizumab $1.0 \mathrm{mg} / \mathrm{ml}$ container.

\section{Randomization}

Randomization was performed by means of an Excel (Microsoft, Seattle, Wash., USA) calculation randomly producing the numbers ' 1 ' (for group I) and ' 2 ' (for group II), specifying 30 values per group. Consecutive patients at all sites were assigned to their treatment group according to the resulting randomization list.

\section{Statistical Analysis and Sample Size Calculation}

Statistical analysis was performed with the SPSS software version 19.0 (SPSS Inc., Chicago, Ill., USA). For comparison of pa- rameters between groups, the Mann-Whitney U test was used. For comparison of data within groups at different time points, the Wilcoxon test was performed. $\mathrm{p}<0.05$ was considered statistically significant.

The sample size calculation resulted in 23 patients per group. This was based on the following assumptions: a visual improvement of 3 lines was considered a clinically relevant benefit (success) irrespective of baseline visual acuity. Preliminary studies by the authors had resulted in a proportion of $20 \%$ of patients treated with IH alone improving by $\geq 3$ lines $[4,14]$. Of patients treated with VEGF inhibitors alone, a proportion of $>55 \%$ improved by $\geq 3$ lines [9]. Consequently, the present randomized trial should be large enough to reject the null hypothesis of equal success rates in the IH and IH-plus-anti-VEGF groups (significance level, $\alpha=5 \%$ ) with a probability (power) of $80 \%$ if the true success rates are $20 \%$ for $\mathrm{IH}$ and $55 \%$ for IH plus anti-VEGF. Based on the arcsin approximation, at least 23 patients per group were needed to achieve this goal. It was planned that approximately 60 patients would be screened to achieve a sample size of approximately 55 eligible patients within an estimated 12 -month recruitment period. It was also expected that, of these patients, approximately 50 would complete the treatment through week 52 , i.e. the time point for the secondary endpoint without major protocol violations.
10

Ophthalmologica 2015;233:8-17 DOI: $10.1159 / 000369566$
Kreutzer et al. 
Table 2. Patient characteristics in the RAVO study

\begin{tabular}{|c|c|c|c|}
\hline & Group I & Group II & $\mathrm{p}^{*}$ \\
\hline Patients, $\mathrm{n}$ & 28 & 30 & \\
\hline Age (min./max.), years & $61.0(21 / 88)$ & $60.9(34 / 87)$ & 0.467 \\
\hline Gender (male/female), n & $21 / 7$ & $14 / 16$ & 0.028 \\
\hline Eye (OD/OS), n & $9 / 19$ & $22 / 8$ & 0.002 \\
\hline Duration of symptoms, weeks & 2.93 & 2.0 & 0.022 \\
\hline \multicolumn{4}{|l|}{ Visual acuity, ETDRS letters } \\
\hline Baseline & 32.4 & 38.3 & 0.304 \\
\hline 2 months & 53.3 & 43.7 & 0.327 \\
\hline 6 months & 56.8 & 63.0 & 0.311 \\
\hline 12 months & 60.5 & 62.6 & 0.649 \\
\hline \multicolumn{4}{|l|}{$\mathrm{CRT}, \mu \mathrm{m}$} \\
\hline Baseline & 725 & 616 & 0.125 \\
\hline 2 months & 424 & 576 & 0.024 \\
\hline 6 months & 367 & 290 & 0.148 \\
\hline 12 months & 278 & 251 & 0.666 \\
\hline \multicolumn{4}{|l|}{ Hematocrit, vol\% } \\
\hline Baseline & 40.3 & 42.6 & 0.123 \\
\hline 2 months & 38.2 & 38.8 & 0.510 \\
\hline 6 months & 38.8 & 39.2 & 0.457 \\
\hline 12 months & 38.9 & 39.3 & 0.291 \\
\hline Injections (min./max.) at 12 months, $\mathrm{n}$ & $5.5(1 / 11)$ & $3.2(0 / 9)$ & 0.004 \\
\hline Hemodilutions during first 2 months, $\mathrm{n}$ & 1.5 & 1.8 & 0.08 \\
\hline
\end{tabular}

Values apart from number of patients, gender and eye are expressed as means. OD = Right eye; OS = left eye; min. = minimum; max. = maximum. ${ }^{*}$ Mann-Whitney $U$ test.

\section{Results}

Study recruitment was terminated when 58 patients were included: 28 patients in group I and 30 patients in group II. Up to that point, no dropout or loss to follow-up had occurred within the study period. All patients but 1 in group II completed the full 12-month study. This patient dropped out after 6 months of follow-up because he moved abroad for work reasons. Data on this patient were included in the intention-to-treat analysis using the lastobservation-carried-forward method. Table 2 shows the patient characteristics for both groups. No significant differences were found for visual acuity at baseline and for age (table 2). A significant difference was found for laterality of the affected eye and gender distribution between the groups. Another significant difference was found for mean duration of symptoms, which was 2.9 weeks (min. 1.0, max. 7.0) in group I and 2.0 weeks (min. 1.0, max. 7.0) in group II $(\mathrm{p}=0.022)$. Though eyes in group I on average had slightly worse baseline ETDRS letter scores at 32.4 $( \pm 21.1)$ letters compared to eyes in group II with 38.3 $( \pm 24.6)$ letters at baseline, no statistical significance was found ( $\mathrm{p}=0.304)$ (table 2; fig. 1a).

IV Ranibizumab vs. IH in the Treatment of Macular Edema Secondary to CRVO

\section{Visual Acuity}

At the end of month 2 (i.e. the primary endpoint, at which, in group II, IVI of ranibizumab $0.5 \mathrm{mg}$ could be initiated) ETDRS letter scores in group I eyes had significantly improved to $53.3( \pm 18.9)$ letters $(\mathrm{p}<0.001)$ compared to only a slight improvement in group II eyes with $43.7( \pm 28.5)$ letters $(p=0.030)$. Nonetheless, statistical analysis detected no significant difference in visual acuity between the groups at month $2(\mathrm{p}=0.279)$. Looking at the mean change in letters (ETDRS) after 2 months of treatment, the eyes showed an increase of $+20.9( \pm 21.4)$ letters in group I compared to +5.4 $( \pm 19.4)$ letters in group II $(\mathrm{p}=0.006)$ (fig. 1b). At month 6 , the mean visual acuity was $56.8( \pm 23.0)$ letters in group I compared to $63.0( \pm 19.7)$ in group II, and the mean changes in letters were $+24.4( \pm 20.1$, group I) and +24.7 ( \pm 18.4 , group II; $p=0.957)$. At the secondary endpoint at 12 months, group I showed a mean ETDRS letter score of $60.5( \pm 22.9)$ letters compared to 62.6 $( \pm 22.3)$ letters in group II $(\mathrm{p}=0.649)$, and the mean change in visual acuity was $+28.1( \pm 19.3)$ letters in group I compared to $+25.2( \pm 20.9)$ letters in group II $(p=0.326)$. An improvement of $\geq 3$ lines ( $\geq 15$ letters $)$ 
Fig. 1. a Mean ETDRS letter score $( \pm S E)$ in group I (IH with prompt IVI of ranibizumab $0.5 \mathrm{mg}$ ) and group II (IH with IVI of ranibizumab $0.5 \mathrm{mg}$ deferred for 2 months). b Mean change in best-corrected visual acuity in ETDRS letters $( \pm$ SE) compared to baseline in group I and group II.

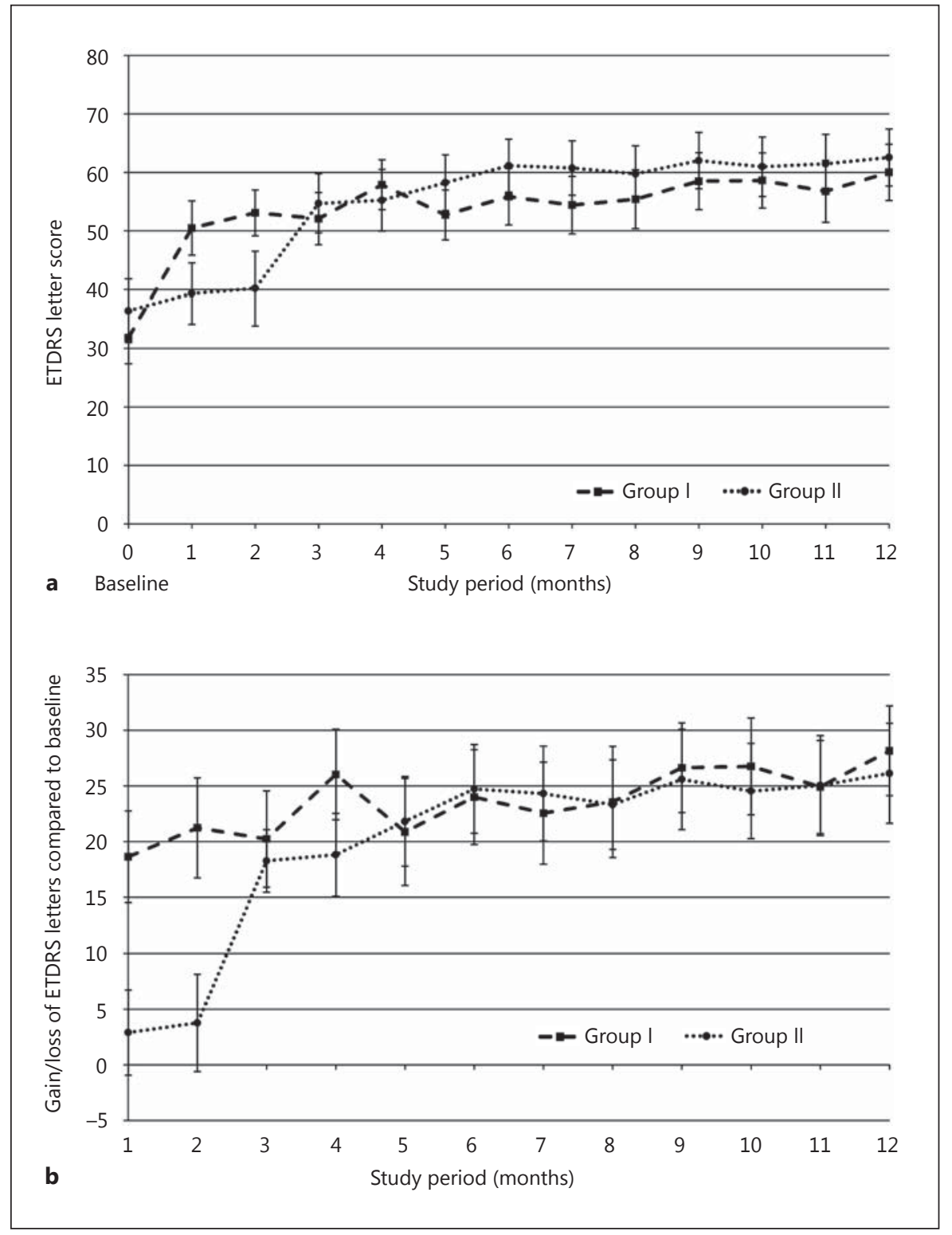

at 12 months was found in 21 of the 28 eyes $(75 \%)$ in group I and in 20 of the 30 eyes $(67 \%)$ in group II ( $\mathrm{p}=$ $0.570, \chi^{2}$ test).

\section{Central Retinal Thickness}

The mean CRT at baseline was $725( \pm 258) \mu \mathrm{m}$ in group I compared to $616( \pm 227) \mu \mathrm{m}$ in group II $(\mathrm{p}=0.125)$ (fig. 2a). After 2 months of treatment with IH and IVI, the mean CRT decreased to $424( \pm 268) \mu \mathrm{m}$ in group I, while $\mathrm{IH}$ alone caused only a small decrease to $576( \pm 312) \mu \mathrm{m}$ in group II $(\mathrm{p}=0.024)$. The change in CRT was -301 $( \pm 298) \mu \mathrm{m}$ in group I compared to $-40( \pm 335) \mu \mathrm{m}$ in group II ( $p=0.002)$ (fig. $2 b)$. At 6 months, after possible PRN initiation of ranibizumab treatment in group II eyes, the mean CRT measured in group I was $367( \pm 199) \mu \mathrm{m}$ compared to $290( \pm 111) \mu \mathrm{m}$ in group II $(\mathrm{p}=0.148)$. There were comparable decreases in mean CRT of $-358( \pm 290)$ $\mu \mathrm{m}$ and $-326( \pm 244) \mu \mathrm{m}$ at 6 months in group I and group II, respectively $(\mathrm{p}=0.779)$. At month 12 (the secondary endpoint), in group I eyes, the mean CRT had decreased to $278( \pm 139) \mu \mathrm{m}$ compared to $251( \pm 87) \mu \mathrm{m}$ in group II eyes $(\mathrm{p}=0.666)$. The mean reductions of CRT compared to baseline were $-447( \pm 268) \mu \mathrm{m}$ in group I and -359 $( \pm 226) \mu \mathrm{m}$ in group II $(\mathrm{p}=0.257)$. 
Fig. 2. a Mean CRT $( \pm$ SE) in group $I(I H$ with prompt IVI of ranibizumab $0.5 \mathrm{mg}$ ) and group II (IH with IVI of ranibizumab $0.5 \mathrm{mg}$ deferred for 2 months). b Mean change in CRT $( \pm$ SE) compared to baseline in group I and group II.

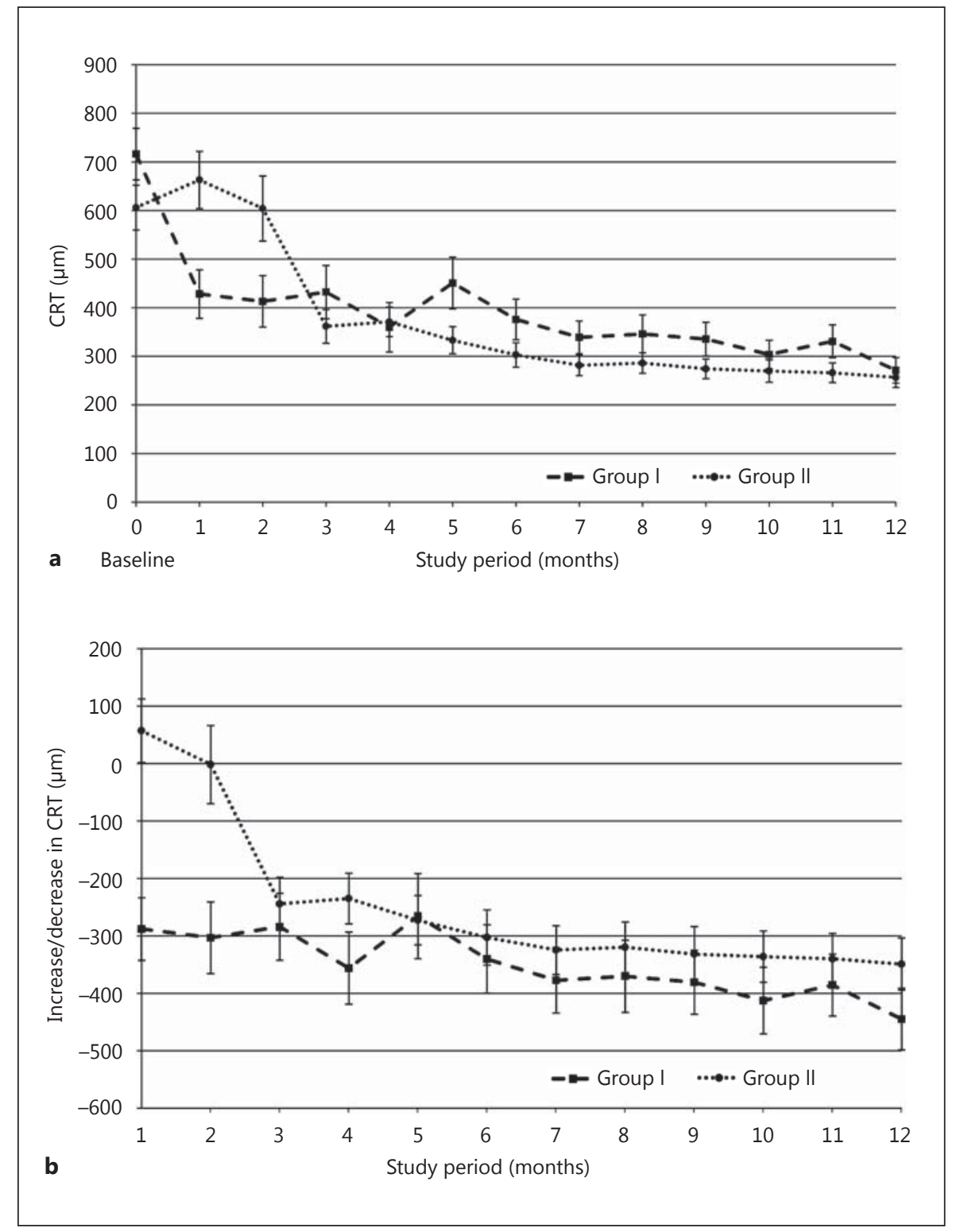

Rheologic Outcome and Number of IVI

During the first 2, months both groups received mandatory $\mathrm{IH}$ in order to decrease the hematocrit levels for better perfusion. No complications were noted due to IH. The baseline hematocrit levels were 40.3 vol\% (group I) and 42.6 vol\% (group II; $\mathrm{p}=0.123$ ). At month 2 , the average hematocrit levels in group I had decreased to 38.2 vol\% compared to $38.8 \mathrm{vol} \%$ in group II $(\mathrm{p}=0.510)$. In both groups, the reduction in hematocrit levels was statistically significant ( $\mathrm{p}=0.010$ and $\mathrm{p}<0.001$, respectively; Wilcoxon test).

The mean number of IVI after 12 months was significantly higher in group I with 5.5 IVI (min. 1, max. 11) than in group II with 3.2 IVI ( $\min .0$, max. 9; $p=0.003$ ). Considering the earlier initiation of IVI of ranibizumab $0.5 \mathrm{mg}$ in group I, we compared the amount of injections up to month 10 in group I with the number of IVI performed from month 2 up to month 12 in group II. The result was consistent with the primary calculation with 5.2 IVI (min. 1, max. 9) in group I compared to 3.2 IVI (min. 0, max. 9) in group II ( $\mathrm{p}=0.006)$. In group II, in which intravitreal treatment with ranibizumab could be initiated after 2 months, 10 of the 30 patients (30\%) did not receive any ranibizumab treatment during the whole study period ( $\mathrm{p}=0.001, \chi^{2}$ test). 
Table 3. Adverse events (n) in the RAVO study

\begin{tabular}{lll}
\hline & $\begin{array}{l}\text { Group I } \\
(\mathrm{n}=27)\end{array}$ & $\begin{array}{l}\text { Group II } \\
(\mathrm{n}=27)\end{array}$ \\
\hline Iris neovascularization & $1(4 \%)$ & $1(4 \%)$ \\
Retinal detachment & 0 & 0 \\
Endophthalmitis & 0 & 0 \\
Episode of depression & $1(4 \%)$ & 0 \\
Ischemic stroke & 0 & 0 \\
Angina pectoris & 0 & 0 \\
Myocardial infarction & 0 & 0 \\
\hline
\end{tabular}

\section{Adverse Events}

Adverse events are summarized in table 3. No serious ocular and systemic adverse events occurred during the study period. One eye in each group developed rubeosis iridis and was treated with panretinal photocoagulation. The neovascularization of the iris dissolved in both cases.

\section{Discussion}

The role of VEGF in the pathogenesis of macular edema secondary to CRVO has been postulated on the basis of raised protein levels in vitreous gel samples of eyes with CRVO [15]. Early treatment strategies used bevacizumab to reduce the amount of VEGF in venous occlusive disease $[9,11,16]$. Ranibizumab, a monoclonal antibody against VEGF with good retinal penetration, had been primarily developed for the treatment of neovascular age-related macular degeneration $[17,18]$ and later proved effective for the treatment of macular edema following CRVO [12, 19]. Ranibizumab aims at reducing the effects of complications succeeding the venous occlusion. Whether it increases the likelihood of reperfusion remains unclear. According to the German guidelines for the therapy of macular edema after retinal vein occlusion, patients were treated with $\mathrm{IH}$ to enhance venous perfusion by increasing blood viscosity. Though it would have been more appropriate to compare the treatment group with ranibizumab to a group without any anti-VEGF treatment, based on the initial experiences with bevacizumab at the start of the trial, the authors considered such a protocol unethical, and it would also have been impossible to recruit a full study population as, predictably, there would have been high dropout rates from a group without intravitreal treatment.
Therefore, a possibility for delayed anti-VEGF treatment was built into the protocol at month 2 for group II patients.

In the RAVO trial, we found a significant difference between IH in combination with prompt IVI of ranibizumab $0.5 \mathrm{mg}$ (group I) and IH with IVI deferred until month 2 (group II). The mean letter gains after 4 and 8 weeks were significantly higher in group I than group II eyes $(p<0.001$ each). In accordance with this, a significantly higher reduction of CRT was found by OCT in group I at months 1 and 2 compared to group II ( $p<0.001$ each). IH plus IVI of ranibizumab resulted in a fast increase in visual acuity via a rapid reduction of macular edema (group I). In group II, where only IH was performed during the first 2 months, on average a lesser but still significant increase in visual acuity was found at both months (month $1, \mathrm{p}=0.049$; month $2, \mathrm{p}=0.030$ ) but with no significant decrease in macular edema $(\mathrm{p}=0.147$ and $p=0.673$, respectively).

Interestingly, the benefit achieved in group I eyes compared to that in group II during the first 2 months tapered off when, in group II, for eyes with persistent macular edema at month 2 , IVI treatment with ranibizumab was initiated if needed. At months 3-12, no significant difference concerning visual acuity and macular edema could be found between the two groups. The same applied to the amount of letters gained on the ETDRS chart as well as the reduction of macular edema. Only at month 4 did group I eyes show a significantly higher gain in letters compared to group II eyes ( $\mathrm{p}=$ 0.049 ). From month 5 onwards, eyes in group II even showed slightly better ETDRS letter scores than eyes in group I. This may be due to recurrences of macular edema in some eyes in group I. In group II, on the other hand, at 5 months most eyes that needed it were given an intravitreal ranibizumab upload treatment, which resulted in a lower percentage of eyes with variability in visual acuity.

Compared to IH alone, combined treatment with ranibizumab showed an enhanced effect on visual acuity recovery and the reduction of macular edema in the initial phase of the study (first 2 months). Though we did not compare ranibizumab treatment alone to only hemodilution, one can assume that the effect in group I may be due to ranibizumab. In group II, we could also find a significant effect on visual acuity but no significant reduction of macular edema. On the other hand, a noteworthy one third of eyes in group II recovered from their CRVO with IH treatment alone and without further need for IVI during the whole study period. In fact, the delayed introduc- 
tion of ranibizumab treatment - at least in our cohort of rather new and previously untreated cases - did not result in a less favorable outcome after 12 months, at least with a 2-month delayed initiation of IVI treatment. In contrast, the ROCC trial showed a marked decline in visual acuity in the no-treatment group during the first 2 months [20]. In our cohort, group II eyes with IH gained some letters within this period.

Additionally, in our study, the delayed anti-VEGF treatment but early IH resulted in a significantly lower need for ranibizumab injections at 12 months and, consequentially, reduced treatment costs. Our results for the effectiveness of hemodilution in CRVO therapy confirm data from two randomized, controlled trials by GlacetBernard et al. $[5,6]$.

No significant differences in complications were found between the two groups. In 1 patient each, panretinal laser photocoagulation was needed in order to treat neovascularization of the iris. In this study, the treatment success was considerable compared to earlier and recent investigations. Eyes in the RAVO study reached a mean final visual acuity of 60.5 ETDRS letters in group I and 62.6 letters in group II. An increase of $\geq 3$ lines from baseline to 12 months was found in $75 \%$ (group I) and 67\% (group II) of the eyes, respectively.

Epstein et al. [21,22], in a randomized, controlled trial evaluating the effectiveness of intravitreal bevacizumab $1.25 \mathrm{mg}$ every 6 weeks for the treatment of CRVO, found gains of $\geq 3$ lines in $60 \%$ of the eyes at 6 and 12 months, respectively, in their treatment group.

In the CRUISE trial, evaluating a $0.3-$ and a $0.5-\mathrm{mg}$ intravitreal regimen with ranibizumab versus sham treatment, Brown et al. [12] and Campochiaro et al. [23] reported a mean visual acuity increase of 12.7 and 14.9 letters after 6 months, respectively, in the treatment groups, and of 13.9 letters in both groups at 12 months. The percentages of patients gaining $\geq 3$ lines on the ETDRS chart were 22 and 27\%, respectively, at 6 months, and 47 and $51 \%$, respectively, at 12 months.

In the ROCC trial, comparing an intravitreal treatment regimen of $0.5 \mathrm{mg}$ of ranibizumab to sham treatment, eyes in the treatment group showed a mean visual acuity gain of 12 letters after 6 months [20]. Reasons for the remarkable results in the RAVO study may be the early initiation of treatment, because only CRVO cases with a duration of symptoms of no more than 8 weeks were included. This finding may support early treatment regimens in CRVO in general. Additionally, the rate of ischemic retinal vein occlusion was very low in our study population, and patients with previous treatment were

IV Ranibizumab vs. IH in the Treatment of Macular Edema Secondary to CRVO excluded. Another reason may be that the strict exclusion criteria for systemic illnesses based on the IH recommendations on average resulted in a systemically healthy patient group. Progressive heart disease, uncontrolled arterial hypertension as well as previous fortified anticoagulation treatment were exclusion criteria.

The disadvantage of delayed treatment has been a major concern in CRVO. In the CRUISE study, eyes treated with ranibizumab $0.5 \mathrm{mg}$ showed a gain of $\geq 3$ lines in $51 \%$ of the eyes after 12 months compared to only $33 \%$ of those eyes for which treatment was initiated with a delay of 6 months [23]. Epstein et al. [22] also detected a less favorable outcome if intravitreal bevacizumab treatment was delayed for 6 months (60\% with bevacizumab vs. 33\% with sham/bevacizumab). In an early prospective case series with intravitreal bevacizumab treatment, we found no significantly reduced visual acuity prognosis within a 3-month time period of delayed therapy initiation [9]. In the RAVO study, a 2-month delay of intravitreal ranibizumab treatment did not result in reduced visual acuity results in early CRVO cases, at least if IH was performed.

From the findings of this study, it may be considered to initiate treatment with $\mathrm{IH}$ in early CRVO subjects without major health risks, especially if there is no early access to intravitreal treatment with ranibizumab. It also seems feasible for patients anxious about intravitreal drug therapy to start treatment with $\mathrm{IH}$ and initiate intravitreal ranibizumab treatment after 6-8 weeks if necessary. This would allow detection of a spontaneous response to $\mathrm{IH}$ and give these patients time to adapt to the thought of IVI. In the RAVO study, IH with delayed intravitreal ranibizumab treatment reduced the amount of necessary IVI over the 12-month period and did not result in a worse visual acuity outcome after 12 months.

During the first year of the CRUISE study, an average of 8.8 injections were given in the ranibizumab $0.5-\mathrm{mg}$ treatment arm [23]. In the ROCC study, an average of 4.3 injections of ranibizumab were needed during a 6-month period [20]. In order to reduce the amount of injections needed, laser photocoagulation of ischemic peripheral retinal areas has been discussed. However, a study by Spaide [24] showed no significant effect on the number of ranibizumab injections. In the RAVO study, 5.5 and 3.2 IVI of ranibizumab $0.5 \mathrm{mg}$ were needed during the 12 -month period. Though this study was not designed to answer this question, further evaluation might be necessary with regard to whether additional IH might have a beneficial effect on the increase in perfusion and the decrease in VEGF release, thus reducing the likelihood of extensive anti-VEGF treatment. 
To note, at baseline, patients in group II on average had a significantly shorter duration of symptoms and a tendency towards reduced CRT. Consequently, patients in group II might have had a shorter progression of disease with decreased chances of pathology. Hence, a possible impact on the results in group II eyes - resulting in a reduced number of injections and noninferior visual acuity at 12 months despite delayed treatment with ranibizumab - cannot fully be excluded. However, as there was no statistically significant difference in baseline visual acuity, we believe that the slight difference in the history of CRVO plays a minor role in the final outcomes. Nonetheless, the reduced sample size is still a weak point of the study.

In summary, in the RAVO study, $\mathrm{IH}$ with prompt and deferred intravitreal treatment with ranibizumab $0.5 \mathrm{mg}$ for early CRVO resulted in high rates of increase in visual acuity over a 12-month period. IH alone resulted in visual acuity recovery in about one third of the patients. From our data, it seems reasonable to primarily treat patients anxious about intravitreal drug therapy, without major systemic risk factors and with early CRVO with IH initially. If spontaneous recovery cannot be achieved, initiation of intravitreal treatment with ranibizumab $0.5 \mathrm{mg}$ improves the chances of visual acuity recovery.

\section{Acknowledgements}

The following investigators of the RAVO Study Group contributed to this study (in alphabetical order): Martin Dirisamer, MD; Christoph Ehlken, MD; Nicolas Feltgen, MD; Paul Foerster, MD; Lutz Hansen, MD; Christos Haritoglou, MD; Lars-Olaf Hattenbach, MD; Bernd Junker, MD; Anselm Kampik, MD; Thomas C. Kreutzer, MD; Peter Laubichler, MD; Alireza Mirshahi, MD; Amelie Pielen, MD; Siegfried G. Priglinger, MD; Rupert W. Strauss, MD; Michael Ulbig, MD; Armin Wolf, MD.

The study was in part financially supported by Novartis Pharma GmbH, Nuremberg, Germany.

\section{Disclosure Statement}

A. Pielen is a paid consultant for Allergan, Bayer HealthCare and Novartis. N. Feltgen has received funds from Novartis, Allergan, Bayer HealthCare and Heidelberg Engineering.

\section{References}

$\checkmark 1$ Rogers S, McIntosh RL, Cheung N, Lim L, Wang JJ, Mitchell P, Kowalski JW, Nguyen $\mathrm{H}$, Wong TY; International Eye Disease Consortium: The prevalence of retinal vein occlusion: pooled data from population studies from the United States, Europe, Asia, and Australia. Ophthalmology 2010;117:313319.

$\checkmark 2$ Evaluation of grid pattern photocoagulation for macular edema in central vein occlusion. The Central Vein Occlusion Study Group M report. Ophthalmology 1995;102:1425-1433.

-3 A randomized clinical trial of early panretinal photocoagulation for ischemic central vein occlusion. The Central Vein Occlusion Study Group N report. Ophthalmology 1995;102: 1434-1444.

4 Hansen LL, Wiek J, Wiederholt M: A randomised prospective study of treatment of non-ischaemic central retinal vein occlusion by isovolaemic haemodilution. Br J Ophthalmol 1989;73:895-899.

5 Glacet-Bernard A, Zourdani A, Milhoub M, Maraqua N, Coscas G, Soubrane G: Effect of isovolemic hemodilution in central retinal vein occlusion. Graefes Arch Clin Exp Ophthalmol 2001;239:909-914.
6 Glacet-Bernard A, Atassi M, Fardeau C, Romanet JP, Tonini M, Conrath J, Denis P, Mauget-Faÿsse M, Coscas G, Soubrane G, Souied E: Hemodilution therapy using automated erythrocytapheresis in central retinal vein occlusion: results of a multicenter randomized controlled study. Graefes Arch Clin Exp Ophthalmol 2011;249:505-512.

7 Wong TY, Scott IU: Clinical practice. Retinalvein occlusion. N Engl J Med 2010;363:21352144.

8 Hoerauf H, Feltgen N, Bartz-Schmidt KU, Bertram B, Bornfeld N, Hattenbach L-O, Heimann H, Helbig H, Kampik A: Stellungnahme der Deutschen Ophthalmologischen Gesellschaft, der Retinologischen Gesellschaft und des Berufsverbandes der Augenärzte Deutschlands zur Therapie des Makulaödems beim retinalen Venenverschluss: Therapeutische Strategien. DOG Publikationen 2012. http://www.dog.org/ wp-content/uploads/2009/08/Druckversion.pdf.

-9 Priglinger SG, Wolf AH, Kreutzer TC, Kook D, Hofer A, Strauss RW, Alge CS, Kunze C, Haritoglou C, Kampik A: Intravitreal bevacizumab injections for treatment of central retinal vein occlusion: six-month results of a prospective trial. Retina 2007;27:10041012.
10 Stahl A, Agostini H, Hansen LL, Feltgen N: Bevacizumab in retinal vein occlusion-results of a prospective case series. Graefes Arch Clin Exp Ophthalmol 2007;245:1429-1436.

11 Stahl A, Struebin I, Hansen LL, Agostini HT, Feltgen N: Bevacizumab in central retinal vein occlusion: a retrospective analysis after 2 years of treatment. Eur J Ophthalmol 2010;20: 180-185.

12 Brown DM, Campochiaro PA, Singh RP, Li Z, Gray S, Saroj N, Rundle AC, Rubio RG, Murahashi WY; CRUISE Investigators: Ranibizumab for macular edema following central retinal vein occlusion: six-month primary end point results of a phase III study. Ophthalmology 2010;117:1124-1133.

13 Winter V, Gille J, Richter A, Sablotzki A, Wiedemann B: Preoperative hypervolemic hemodilution with $6 \%$ hydroxyethyl starch $130 / 0,4$ (HES 130/0.4) solution as a way of reducing needs for donor blood transfusion (in Russian). Anesteziol Reanimatol 2006;2:43-47.

14 Hansen LL, Wiek J, Schade M, Müller-Stolzenburg N, Wiederholt N: Effect and compatibility of isovolaemic haemodilution in the treatment of ischaemic and non-ischaemic central retinal vein occlusion. Ophthalmologica 1989;199:90-99. 
15 Ehlken C, Rennel ES, Michels D, Grundel B, Pielen A, Junker B, Stahl A, Hansen LL, Feltgen N, Agostini HT, Martin G: Levels of VEGF but not $V_{E G F}$ 165b are increased in the vitreous of patients with retinal vein occlusion. Am J Ophthalmol 2011;152:298-303. e1.

16 Kreutzer TC, Alge CS, Wolf AH, Kook D, Burger J, Strauss R, Kunze C, Haritoglou C, Kampik A, Priglinger S: Intravitreal bevacizumab for the treatment of macular oedema secondary to branch retinal vein occlusion. $\mathrm{Br}$ J Ophthalmol 2008;92:351-355.

17 Ferrara N, Damico L, Shams N, Lowman H, Kim R: Development of ranibizumab, an antivascular endothelial growth factor antigen binding fragment, as therapy for neovascular age-related macular degeneration. Retina 2006;26:859-870
18 Heier JS, Antoszyk AN, Pavan PR, Leff SR, Rosenfeld PJ, Ciulla TA, Dreyer RF, Gentile RC, Sy JP, Hantsbarger G, Shams N: Ranibizumab for treatment of neovascular age-related macular degeneration: a phase I/II multicenter, controlled, multidose study. Ophthalmology 2006;113:633.e1-e4.

19 Heier JS, Campochiaro PA, Yau L, Li Z, Saroj N, Rubio RG, Lai P: Ranibizumab for macular edema due to retinal vein occlusions: longterm follow-up in the HORIZON trial. Ophthalmology 2012;119:802-809.

20 Kinge B, Stordahl PB, Forsaa V, Fossen K, Haugstad M, Helgesen OH, Seland J, SteneJohansen I: Efficacy of ranibizumab in patients with macular edema secondary to central retinal vein occlusion: results from the sham-controlled ROCC study. Am J Ophthalmol 2010;150:310-314.
1 Epstein DL, Algvere PV, von Wendt G, Seregard S, Kvanta A: Bevacizumab for macular edema in central retinal vein occlusion: a prospective, randomized, double-masked clinical study. Ophthalmology 2012;119:1184-1189.

22 Epstein DL, Algvere PV, von Wendt G, Seregard S, Kvanta A: Benefit from bevacizumab for macular edema in central retinal vein occlusion: twelve-month results of a prospective, randomized study. Ophthalmology 2012;119:2587-2591.

23 Campochiaro PA, Brown DM, Awh CC, Lee SY, Gray S, Saroj N, Murahashi WY, Rubio RG: Sustained benefits from ranibizumab for macular edema following central retinal vein occlusion: twelve-month outcomes of a phase III study. Ophthalmology 2011;118:2041-2049.

24 Spaide RF: Prospective study of peripheral panretinal photocoagulation of areas of nonperfusion in central retinal vein occlusion. Retina 2013;33:56-62. 